\title{
Optical And Electrical Properties Of TiOPc Doped Alq Thin Films
}

\author{
M. Ramar, C. K. Suman", Priyanka Tyagi and R. Srivastava \\ CSIR-Network of Institutes for Solar Energy \\ CSIR - National Physical Laboratory, Dr. K. S. Krishnan Marg, New Delhi -110012, India \\ *E-mail:sumanck@nplindia.org
}

\begin{abstract}
The Titanyl phthalocyanine (TiOPc) was doped in Tris (8-hydroxyquinolinato) aluminum ( $\mathrm{Alq}_{3}$ ) with different concentration. The thin film of optimized doping concentration was studied extensively for optical and electrical properties. The optical properties, studied using ellipsometry, absorption and photoluminescence. The absorption peak of $\mathrm{Alq}_{3}$ and TiOPc was observed at $387 \mathrm{~nm}$ and $707 \mathrm{~nm}$ and the photo-luminescence intensity (PL) peak of doped thin film was observed at $517 \mathrm{~nm}$. The DC and AC electrical properties of the thin film were studied by current density-voltage $(J-V)$ characteristics and impedance over a frequency range of $100 \mathrm{~Hz}-1 \mathrm{MHz}$. The electron mobility calculated from trap-free space-charge limited region (SCLC) is $0.17 \times 10^{-5} \mathrm{~cm}^{2} / \mathrm{Vs}$. The Cole-Cole plots shows that the TiOPc doped $\mathrm{Alq}_{3}$ thin film can be represented by a single parallel resistance $R_{P}$ and capacitance $C_{P}$ network with a series resistance $R_{S}(10 \Omega)$. The value of $R_{P}$ and $C_{P}$ at zero bias was $1587 \Omega$ and $2.568 \mathrm{nF}$ respectively. The resistance $R_{P}$ decreases with applied bias whereas the capacitance $C_{P}$ remains almost constant.
\end{abstract}

Keywords: Thin film, Impedance, Electron mobility, Doped.

PACS: 73,78 and 85.

\section{INTRODUCTION}

Recently, organic semiconductors have become very attractive materials capable of replacing inorganic semiconductors in the development of low-cost, large area and light weight optoelectronic devices, such as organic light emitting diodes (OLEDs), organic solar cells (OSCs) and organic thin film transistors (OTFTs) [1-4]. The doping in organic semiconductor plays a vital role in tuning the optical and electrical properties [5]. The interface between organic semiconductors and metals are also dependent on the doping concentration. The carrier injection in the device is influenced by suitable dopant in the organic semiconductor that leads to the enhancement of device life time [6]. Now a days, there is a new trend in doping of organic semiconductor for reducing the contact resistance with metal and lowering the energy barrier at the interface due to band bending [7]. Since $\mathrm{Alq}_{3}$ is the widely used electron transport material as well as green emissive material, therefore it is important to study the optical and electrical properties of doped $\mathrm{Alq}_{3}$.

In this work we have discussed about the optical and electrical properties of TiOPc doped $\mathrm{Alq}_{3}$ thin films using current density-voltage $(J-V)$ characteristics and impedance spectroscopy in the broad frequency range $100 \mathrm{~Hz}-1 \mathrm{MHz}$.

\section{EXPERIMENTAL METHODS}

The electron only devices (EOD) were fabricated on glass substrates. In the structure of $\mathrm{Al} /$ organic/Al, EOD composed of a $100 \mathrm{~nm}$ thick of Titanyl phthalocyanine (TiOPc) doped Tris(8hydroxyquinolinato) aluminum $\left(\mathrm{Alq}_{3}\right)$, Aluminium (Al) $(100 \mathrm{~nm})$ were used for the anode and cathode both, respectively. The optimized doping concentration of TiOPc was $2 \%$ by weight.

Current density-voltage $(J-V)$ characteristics of EOD were measured with a programmable Keithley 2400 power source. Impedance spectroscopy (IS) measurement was performed using Impedance/GainPhase Analyzer (Solartron, model SI 1260) System. A $100 \mathrm{mV}$ amplitude AC signal superimposed on a DC bias was used to measure the device Impedance as a function of $\mathrm{AC}$ frequency and DC bias.

\section{RESULTS AND DISCUSSION}

The surface morphology of TiOPc doped $\mathrm{Alq}_{3}$ thin 
film was studied by atomic force microscopy (AFM). Figure .1 shows AFM image of TiOPc doped $\mathrm{Alq}_{3}$ thin film. The root mean square (RMS) value of roughness for the thin film was found $0.5742 \mathrm{~nm}$. Due to doping of TiOPc the spikes appeared in crystalline form on the thin film. That spikes dominates in charge transport mechanism.

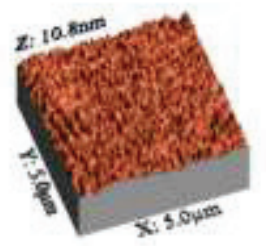

FIGURE 1. AFM image of TiOPc doped $\mathrm{Alq}_{3}$ thin film.

The optical properties of the thin film were studied by absorption, photoluminescence (PL) and ellipsometry spectroscopy. Figure 2 (a) shows the absorption and PL spectrum in the wavelength range of $200-1000 \mathrm{~nm}$. The maximum absorption was observed at a wavelength of $387 \mathrm{~nm}$ and $707 \mathrm{~nm}$ which corresponds to $\mathrm{Alq}_{3}$ and $\mathrm{TiOPc}$, respectively. The photo-luminescence intensity (PL) peak was observed at $517 \mathrm{~nm}$. The absorption and PL both show the broad spectrum in the vicinity of the above mentioned peak. The ellipsometric response of the thin film was measured in the $200-1000 \mathrm{~nm}$ spectral range at an incident angle of $50^{\circ}$. The refractive index (n) and extinction coefficient $(\mathrm{k})$ spectra are shown in figure 2 (b).

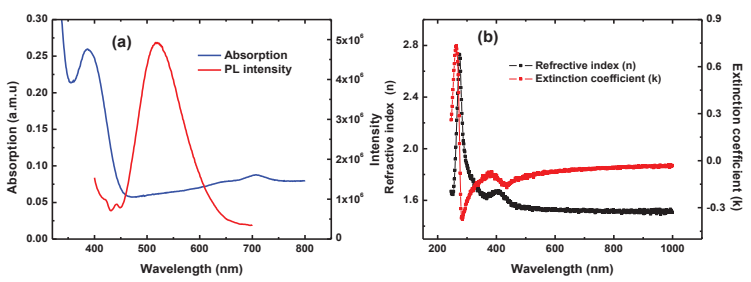

FIGURE 2. (a) Absorption and Photoluminescence (PL) Intensity spectra of $\mathrm{TiOPc}$ doped $\mathrm{Alq}_{3}$ thin film and (b) Refractive index (n) and Extinction coefficient (k) as a function of wavelength

The index of refraction are found between $1.5-2.7$ whole wavelength range. These $\mathrm{n}$ values are higher than the typical organic semiconductor used as electron transport layer. The extinction coefficient $(\mathrm{k})$ is 0.72 at wavelengths of $265 \mathrm{~nm}$ which corresponds to an absorption coefficient $\alpha \sim 3.8 \times 10^{2} \mathrm{~cm}^{-1}$.

The $J-V$ characteristics of the electron only device are shown in figure 3 . Two regions are clearly seen in figure. The slope of double logarithmic $J-V$ plot in low voltage region is 1 , corresponding to an Ohmic conduction mechanism and the slope of higher voltage region is $\sim 2$, which is a sign of space-charge-limited current (SCLC) with exponential trap distributions. This indeed indicates the significant Ohmic injection of electrons in the fabricated electron only device. To calculate the electron mobility in the trap-free SCLC region, we use the Mott-Gurney equation for trap-free SCLC [8].

$$
I=\frac{9}{8} \mu \varepsilon \varepsilon_{0} \frac{v^{2}}{d^{3}}
$$

Where $\varepsilon$ is the dielectric constant, $\varepsilon_{0}$ is the permittivity of free space, $\mu$ is the carrier mobility, $v$ is the applied voltage, $d$ is the active layer thickness and $J$ is the current density. At high applied bias voltage, current density was found to follow Eq. (1), which could be ascribed as the field and charge carrier density dependence of the mobility. The results show that the electron mobility in the TiOPc doped $\mathrm{Alq}_{3}$ is $\sim 0.17 \times 10^{-5} \mathrm{~cm}^{2} / \mathrm{Vs}$. The electron mobility of TiOPc doped $\mathrm{Alq}_{3}$ device is of four order compare to $\mathrm{Alq}_{3}$ (mobility $\sim 0.9 \times 10^{-9} \mathrm{~cm}^{2} / \mathrm{Vs}$ ) device.

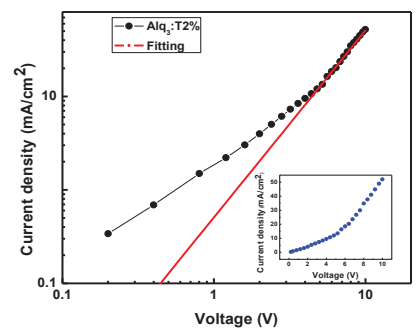

FIGURE 3. Log - log plot of current density - voltage $(J-V)$ characteristics of electron-only device. Inset figure shows the normal $J-V$ characteristics.

Figure 4. Show the frequency dependence of the real $(\operatorname{Re} Z)$ and imaginary $(\operatorname{Im} Z)$ parts of impedance at different bias voltages. The complex impedance $Z(f)$, can be represented as a function of frequency as,

$$
z(f)=Z^{\prime}(f)+Z^{\prime \prime}(f)
$$

Where Z' and Z" are the real and imaginary parts of impedance. It is observed that the magnitude of $\operatorname{Re} Z$ are constant with frequency up to $10 \mathrm{kHz}$ and it decreases with frequency between $10 \mathrm{kHz}$ to $1 \mathrm{MHz}$. The magnitude of $\operatorname{Re} Z$ decreases with applied bias voltages but for all voltage the value of $\mathrm{Re} Z \mathrm{Z}$ merges in the higher frequency region at above $50 \mathrm{kHz}$. The curves also display single relaxation process and indicate an increase in ac conductivity with frequency. The $\operatorname{Im} Z$ reaches a maximum peak $\operatorname{Im}(Z)_{\max }$ for all bias voltage at different frequency. The value of Im $(Z)_{\max }$ shifts to higher frequency with applied bias voltage. The frequency corresponding to $\operatorname{Im}(Z)_{\max }$ called relaxation frequency shifts to higher values with increase of applied bias voltage. This is an indication of increasing loss in the materials with increase of applied bias voltage. 

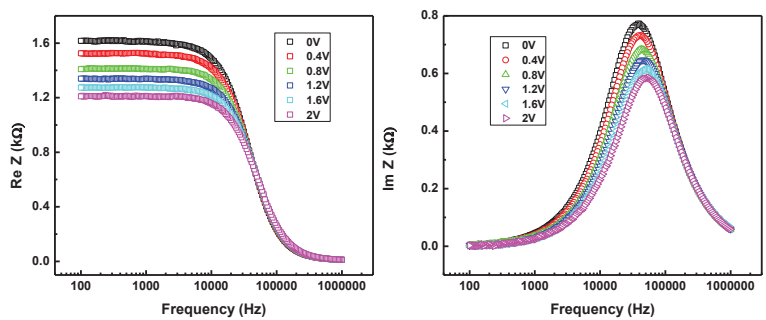

FIGURE 4. Real and imaginary part of impedance as a function of frequency at various applied bias voltages.

The Cole-Cole plots of the Re Z and Im Z at $100 \mathrm{~Hz}$ to $1 \mathrm{MHz}$ for different dc bias voltages at room temperature for the electron only device are shown in figure 5 .

The plot shows a single semicircle at bias voltages 0 $\mathrm{V}$ to $2 \mathrm{~V}$ and the size of the semicircle decreases rapidly as the dc bias voltage increases. Therefore, the TiOPc doped $\mathrm{Alq}_{3}$ device can be modeled as an $\mathrm{RC}$ equivalent electrical circuit of which a contact series resistance $R_{S}$ with a single parallel resistance $\left(R_{P}\right)$ and

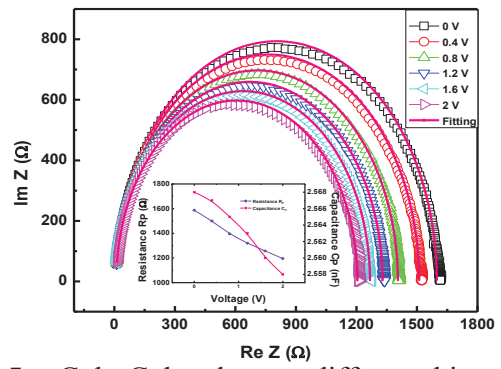

FIGURE 5. Cole-Cole plots at different bias voltages at room temperature. Inset figure shows the variation of bulk resistance $R_{P}$ and capacitance $C_{P}$ with different applied bias voltages.

the capacitance $\left(C_{P}\right)$ network. The impedance of the $\mathrm{RC}$ equivalent electrical circuit can be giving by [9]

$$
\begin{aligned}
& Z=R e Z+i \operatorname{Im} Z \\
& =\left[R_{S}+\frac{R_{P}}{1+\omega^{2} R_{P}^{2} c_{P}^{2}}\right]-i\left[\frac{\omega R_{P}^{2} c_{P}}{1+\omega^{2} R_{P}^{2} c_{P}^{2}}\right]
\end{aligned}
$$

By eliminating the angular frequency, the semicircle of the Cole-Cole plot can be written as

$$
\left[R e Z-\left(R_{S}+\frac{R_{P}}{2}\right)\right]^{2}+(-\operatorname{Im} Z)^{2}=\left(\frac{R_{P}}{2}\right)^{2}
$$

This relation defines a circle centered at $\left(R_{S}+R_{P} / 2,0\right)$ with radius of $R_{P} / 2$. The minimum $\operatorname{Re} Z$ value represents the value of $R_{S}$ with the capacitance and it is about $10 \Omega$ and almost same with the different applied bias voltage. The $R_{S}$ can be considered as originating from the electrode contact. The maximum $\operatorname{Re} Z$ value corresponds to the summation of $R_{S}$ and $R_{P}$ to the capacitance. In figure 5 the symbols are the experimental values and the solid lines are the theoretical results obtained from Eq. (4). There is perfect agreement between the experimental and theoretical impedance results.

The fitting data based on Eq. (4) are shown in inset figure 5. It can be seen that the $C_{P}$ is almost independent on the bias voltage, but the $R_{P}$ decreases as the applied bias voltage increases. Generally, $C_{P}$ is directly related to the intrinsic property of used materials, the independent $C_{P}$ on the bias voltage indicates that the device should be acted as a simple parallel plate capacitor.

The decrease of $R_{P}$ with bias voltage is then due to a large number of injected electrons increase as the bias voltage increases. This also indicates that the effective conductivity of the organic material will increase with the bias voltage.

\section{CONCLUSIONS}

The thin film shows RMS roughness of $0.57 \mathrm{~nm}$ with crystalline spikes as integrated morphology. The absorption peaks are found for both $\mathrm{Alq}_{3}$ and TioPc where as the photoluminescence peak was observed only for $\mathrm{Alq}_{3}$ in our studied range of wavelength. The PL peak of TioPc might be beyond $800 \mathrm{~nm}$. The electron mobility of doped $\mathrm{Alq}_{3}$ is $0.17 \times 10^{-5} \mathrm{~cm}^{2} / \mathrm{Vs}$ in SCLC region which is four times higher than the pristine $\mathrm{Alq}_{3}$. The electron only device of doped $\mathrm{Alq}_{3}$ can be modeled as an equivalent parallel $\mathrm{RC}$ network as observed by Cole-Cole plot. The $R_{P}$ is highly dependent on voltage where as the $C_{P}$ is independent of applied bias.

\section{ACKNOWLEDGMENTS}

The authors gratefully recognize the financial support from the Council of Scientific and Industrial Research (CSIR), India for funding as SRF.

\section{REFERENCES}

1. C. W. Tang and S. A. VanSlyke, Appl. Phys. Lett. 51, 913 (1987).

2. C. W. Tang, Appl. Phys. Lett. 48, 183 (1986).

3. S. Noh, C. K. Suman, Y. Hong, C. H. Lee, J. Appl. Phys. 105, 033709 (2009).

4 M. Ramar, C.K. Suman, R. Manomozhi, R. Ahamed and R. Srivastava, RSC. Adv. 4, 32651 (2014).

5. C.-C. Chang, M. -T. Hsieh, J. -F. Chen, S. -W. Hwang, C. H. Chen, Appl. Phys. Lett. 89, 253504 (2006).

6. D. Grozea, A. Turak, Y. Yuan, S. Han, Z. H. Lu, W. Y. Kim, J. Appl. Phys. 101, 033522 (2007).

7. S. W. Tsang, Z. H. Lu, Y. Tao, Appl. Phys. Lett. 90, 132115 (2007)

8. N. F. Mott, R. W. Gurney, Electronic Processes in Ionic Crystals, Second ed. Oxford University, Oxford, 1948.

9. J. R. Macdonald, Impedance Spectroscopy, Wiley, New York, (1987). 
AIP Conference Proceedings is copyrighted by AIP Publishing LLC (AIP). Reuse of AIP content is subject to the terms at: http://scitation.aip.org/termsconditions. For more information, see http://publishing.aip.org/authors/rights-and-permissions. 